\title{
Implicit Simulations using Messaging Protocols
}

\author{
G.A. Kohring \\ C\&C Research Laboratories, NEC Europe Ltd. \\ Rathausallee 10, 53757 St. Augustin, Germany \\ E-mail: kohring@ccrl-nece.de
}

\begin{abstract}
A novel algorithm for performing parallel, distributed computer simulations on the Internet using IP control messages is introduced. The algorithm employs carefully constructed ICMP packets which enable the required computations to be completed as part of the standard IP communication protocol. After providing a detailed description of the algorithm, experimental applications in the areas of stochastic neural networks and deterministic cellular automata are discussed. As an example of the algorithms potential power, a simulation of a deterministic cellular automaton involving $10^{5}$ Internet connected devices was performed.
\end{abstract}

Keywords: Distributed computing; Parallel Algorithms; Neural Networks; Cellular Automata

\section{Introduction}

Most readers will be familiar with the concept of Moore's law as applied to the computer industry, namely, the density of transistors on a chip and with it the speed of the processors doubles roughly every 18 months. [1] It has held true for more than three decades now and shows all signs of holding at least through the end of this decade. However, most readers will be less familiar with similar growth laws in other areas of computer technology, 
e.g., disk capacity has been doubling roughly every 12 months and network bandwidth has been doubling roughly every 9 months. [2] A mere 15 years ago the Internet backbone ran at $64 \mathrm{Kbits} / \mathrm{s}$, this year it is being upgraded to $40 \mathrm{Gbits} / \mathrm{s}$, an increase of nearly six orders of magnitude. The latter point is of particular interest for computational science, because faster networks enable wide area distributed computing on a scale which was not feasible in the past. However, it also implies that the same questions of efficiency now afflicting the processors in modern PCs will soon become apparent in the communications networks themselves.

As has been often mentioned, [3] most processors are idle or running below their maximum capability most of the time, with enormous wastes of cpu cycles. Projects like SETI@HOME[3] and Condor [4] attempt to harness this excess computing power for performing useful work. SETI@HOME's approach is to hide their calculations behind screen savers which are activated whenever the processor is idle. Condor, on the other hand attempts to make all the PCs and workstations in an organization available for running parallel applications, whereby the load on each PC from a Condor job is adjusted to suit the needs of anyone accessing the PC interactively.

In the same vane, the rapidly increasing bandwidth implies that most of a network's capacity will soon go underutilized most of the time. Hence, the question to be addressed here, is whether or not the idle bandwidth can be tapped for performing useful calculations.

Recently, Barabási et al. examined this problem by utilizing the Hyper Text Transmission Protocol (HTTP) to perform calculations aimed at solving a 2-SAT problem during the act of communicating with web servers. [5] Although their algorithm worked as expected, it has several drawbacks. 1) The HTTP [6, 7] protocol uses connected sockets, which require three messages to be exchanged in order to initiate the connection before any data can be sent. Once connected, their algorithm requires two further message exchange wherein the actual computation is performed. Afterwards another message has to be exchanged to properly disconnect the sockets. In total, six messages are exchanged for each computation step. 2) The HTTP protocol is layered on top of the Transmission Control Protocol (TCP) [8] which is in turn layered upon the Internet Protocol (IP). [9, 10] Since each layer introduces its own overhead, it would be more efficient to use a protocol which is closer to the IP level. 3) The number of HTTP servers is actually quite small compared to the total number of devices connected to the Internet, therefore limiting the application to HTTP servers greatly reduces the pool of devices available for performing useful work.

In this paper we describe a different approach based upon the Internet Control Message Protocol (ICMP)[1]], which is layered directly on top of IP. 
ICMP is a connectionless protocol, meaning only the messages containing the data need to be exchanged. No control messages are required for establishing and ending the connection. Furthermore, ICMP is required to be implemented by every Internet capable device, hence there are many more devices which can respond to ICMP messages than can respond to HTTP requests. The next section describes the general approach in more detail. Section three then discusses a version of the algorithm for stochastic neural network models and section four describes an implementation for a deterministic cellular automaton. We conclude with a discussion on the utility of these approaches and their future viability.

\section{Computing with ICMP}

To understand how messages can be used to perform useful calculations one has to understand how message exchange on the Internet works. The Internet is a so-called packet-switching network, 11] meaning that all messages are broken down into one or more packets called datagrams, whereby the order and meaning of the bytes within the datagram are governed by the various transmission protocols which the applications sending the messages are using. Since the communication channels used to transmit the packets are inherently noisy and unreliable, each networking protocol adds some means, however rudimentary, of checking for corrupted data. In the case of IP or ICMP, this takes the form of the so-called Internet check-sum $\left(I_{s}\right)$. If $I_{s}$ is inserted by the sender into the original packet and then recalculated by the receiver, it is possible to determine whether or not an error has occurred during transmission. The basic idea behind the current proposal is to exploit the calculation of $I_{s}$ to perform useful work in addition to its primary function of checking whether or not the packet is corrupted.

Since the Internet Control Message Protocol is layered on top of IP, an IP/ICMP datagram consists of an IP header with 20-60 bytes of control and routing information, plus the ICMP message itself. Most ICMP message types place severe restrictions on the type of data which can be sent as part of the message, however, the Echo Request and Echo Reply messages do not place any restrictions. Normally, this flexibility is used for example by the ping command to measure bandwidth by measuring the time needed for a datagram of a given size to complete a round-trip between two hosts, or to check for size dependent transmission problems on a network connection.

For our purposes, the bytes of an ICMP Echo Request can be thought of as an array of $N$ 16-bit words as depicted in Fig. 1. Whereby, the first byte of the first word indicates the ICMP message type ( 8 for Echo Request and 0 for 


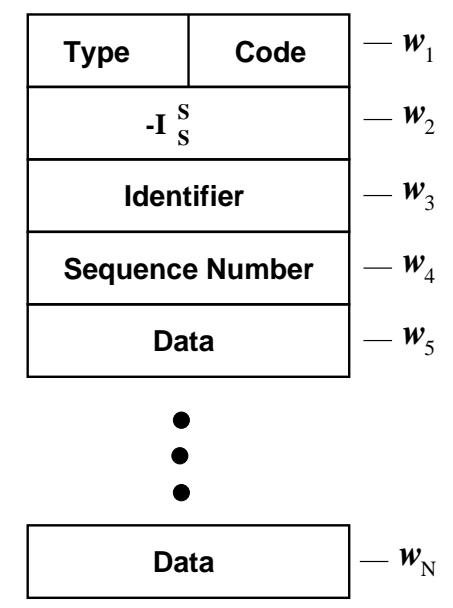

Figure 1: Format of an ICMP Message.

Echo Reply) and the second byte of first word provides room for any special codes associated with the message type (always 0 for Echo Request and Echo Reply). The second word contains the Internet check-sum, $I_{s}^{s}$ for the ICMP part of the datagram, while the third and fourth words contain information to help identify the datagram. The remaining $N-4$ words comprise the data associated with the Echo Request and Echo Reply.

When computing the check-sum the 16-bit words are assumed to represent integers in the one's-complement representation. [12] In a one's-complement representation negative integers are represented by inverting each bit in the representation of their magnitude. Curiously, this leads to two representations for 0 , namely, $0 \ldots 0$ and $1 \ldots 1$, which are designated +0 and -0 respectively. (Contrast this with a two's complement representation used on most computers where there is only one representation for 0 and negative numbers are represented by subtracting one from the magnitude before inverting all the bits.)

One's-complement addition, $\oplus$, is defined as adding two numbers and carrying any overflow bit around to the lowest order bit where it is added to the previous sum. This guarantees that $1 \oplus(-0) \equiv 1$ and not +0 . Furthermore, the addition is circular, i.e., if $M$ is the largest maximum integer, then $M \oplus 1=-M$. With this definition of addition, the Internet check-sum, $I_{s}$, is defined by the equation:

$$
I_{s}=\bigoplus_{i=1}^{N} w_{i},
$$

whereby, the sender sets $w_{2}=0$, for the purpose of calculating the initial 
checksum. To determine whether or not the packet has been corrupted in transit, the receiver computes the function:

$$
F\left(I_{s}\right)=\delta_{k}\left(I_{s},-0\right)
$$

where $\delta_{k}(x, y)=\{1$ if $x=y, 0$ otherwise $\}$ is the Kronecker delta function. If $F\left(I_{s}\right)=1$, the datagram is considered to be uncorrupted. (To understand why $I_{s}=-0$ and not $I_{s}=+0$ indicates an uncorrupted packet, note that $1 \oplus(-1)=-0$.

For checksum purposes a one's complement arithmetic is preferable to a two's compliment because its sensitivity to errors is independent of the bit position. In a two's compliment representation, flipping the most significant bit in an even number of words would yield the same checksum. [12]

Obviously, this algorithm is not completely failsafe, since the probability that any two random configurations will yield the same value of $I_{s}$ is $2^{-16}$. However, it does guard against single bit transmission errors and as these occur on average once in every $2^{10}$ packets, 13 it is sufficient for most applications. Applications requiring a lower error rate need to use a higher level transmission protocol which more error checking.

When the receiver detects a corrupted packet it should simply discard it without emitting an error message to the sender. If the packet is not corrupt, then the receiver should respond to an Echo Request with an Echo Reply. When constructing the Echo Reply message, the receiver takes the original message, replaces the Type byte with 0 for Echo Reply, recomputes the checksum as if it were the sender and sends the entire message back to the original sender, i.e., only $w_{1}$ and $w_{2}$ of the original message are changed. Essentially, then the Echo Request allows us to compute eq. 2 on the remote computer.

Normally, for higher level protocols like FTP, HTTP, etc., the job of calculating the checksum is done automatically by the software which implements the protocol so the user never has to worry about it. However, for low level protocols like ICMP, users are expected to construct the datagram, including $I_{s}$, themselves, which leads us to the possibility of constructing $I_{s}$ in such a way that evaluating eq. 2 has a purpose other than that originally intended one of error checking.

In the definition of the ICMP there are a few small loopholes, [11] e.g., the standards document does not explicitly state that the checksum must be evaluated before answering an Echo Request. In fact, some implementations, which we term non-validating, respond to an Echo Request without bothering to validate the checksum first. In the same vane, the original sender is not explicitly required to validate the Echo Reply response, thus we are at liberty 
to skip this time consuming step. (Strictly speaking both of these actions are incorrect, for if the checksum is wrong, the receiver cannot be certain that the sender really meant to issue an Echo Request, or Echo Reply and not some other ICMP message type. Nevertheless, these "features" will become very useful in what follows.)

\section{Simulating Stochastic Neural Networks}

Unlike HTTP, ICMP is not a reliable protocol, meaning there is no guarantee that a datagram will arrive, or that a reply will be sent, or that replies will be sent in the same order as the requests, or that multiple replies will not be sent for each request or that the requests will even be sent out in the first place. It is the job of a higher level protocol to make these types of guarantees. However, neural networks possess a built-in robustness to noise, which renders these problems practically mute.

For the purposes of demonstrating the algorithm, we use a Hopfield model with limited precision weights. 14, 15, 16] (This is not a very serious restriction because more complicated models can be incorporated using complex neurons with a Hopfield type internal structure.[17])

Very briefly, the model consists of $N$ neurons denoted by $S_{i} \in\{-1,1\}$ and $N^{2}$ couplings denoted by $J_{i j} \in[-L, L]$, where $L$ is the maximum allowed value for each coupling. Typically, $L$ only needs to be a few bits wide (45) for obtaining good results. 16, 15 Finally, the model is endowed with a discrete, time dependent dynamics given by:

$$
S_{i}(t+1)=\operatorname{sign}\left(\sum_{j \neq i}^{N} J_{i j} S_{j}(t)\right) .
$$

The couplings, $J_{i j}$, are created using the clipped Hebb rule:

$$
J_{i j}=B\left(-L, \sum_{\mu=1}^{P} \xi_{i}^{\mu} \xi_{j}^{\mu}, L\right)
$$

where the $\left\{\xi^{\mu}\right\}$, with are the patterns the network should learn and $B(a, x, b)=$ $\{a$ if $x<a, b$ if $x>b, x$ otherwise $\}$

From eq. 3 it is evident that the computationally intensive part of this simulation is the calculation of the sums. By sending an ICMP message to a non-validating IP device, we can induce it to compute this sum for us.

First note that if we set the Identifier and Sequence Number to zero (refer to fig. 1) and recall that in an Echo Reply message both Type and Code are 
zero, then we see that the value of $I_{s}$ returned by the Echo Reply will be simply the sum of the data elements.

For the Echo Request message we can set $I_{s}$ to any arbitrary value since the non-validating IP implementation will just ignore it. The data elements are then set to $J_{i j}$ or $-J_{i j}$ (in one's compliment representation) depending upon the value of $S_{j}(t)$.

Typically, a neural network is updated either sequentially or in parallel, although, Hopfield in his original work used an asynchronous updating algorithm. 14 In this paper we also use an asynchronous updating algorithm since it is a natural fit to the unreliability of IP/ICMP messages mentioned above. After a message is sent a new message is constructed without waiting for a reply from the first message. Once a reply has been received, the corresponding value of $S_{i}$ is immediately updated.

We have tested this algorithm on our laboratory's intranet. As a first step, we selected a subset of devices which are non-validating. None of the workstations or PCs were in this subset, rather it consisted of printers, routers and switches - devices one would not normally think of using for performing numerical simulations. The computer chosen for controlling the simulations was an SGI O2 with a 180 MHz MIPS 5000 processor, while our laboratory's intranet has a peak bandwidth of $100 \mathrm{Mbits} / \mathrm{s}$.

A comparison of the present algorithm versus a standard parallel updating algorithm for the same system size is shown in fig. 2. In these experiments, we intentionally start with an initial configuration at a Hamming distance of 0.25 from a learned pattern and observe how the standard algorithm and the present network algorithm converge towards a stable state. (For the purposes of comparison, one time step in the asynchronous updating algorithm corresponds to $N$ neural updates, while one time step in the parallel updating consists of updating all $N$ neurons.) Evidently, the quality of recall is not adversely affected by the asynchronous updating algorithm nor by the unreliability of the ICMP protocol.

We then tested the same algorithm on a set of randomly chosen devices residing on the world-wide Internet. Our results were similar to those in fig. 2, although the simulation ran much slower because our connection to the Internet has a bandwidth of only $1 \mathrm{Mbit} / \mathrm{s}$. (We will delay a more detailed discussion of the relative performance until section 5.)

These simple proof-of-concept experiments demonstrate that stochastic models can be simulated quite well using only the computational capabilities of the messaging protocols themselves. In the next section we discuss the algorithmic changes need to ensure that deterministic models can also be accurately simulated. 


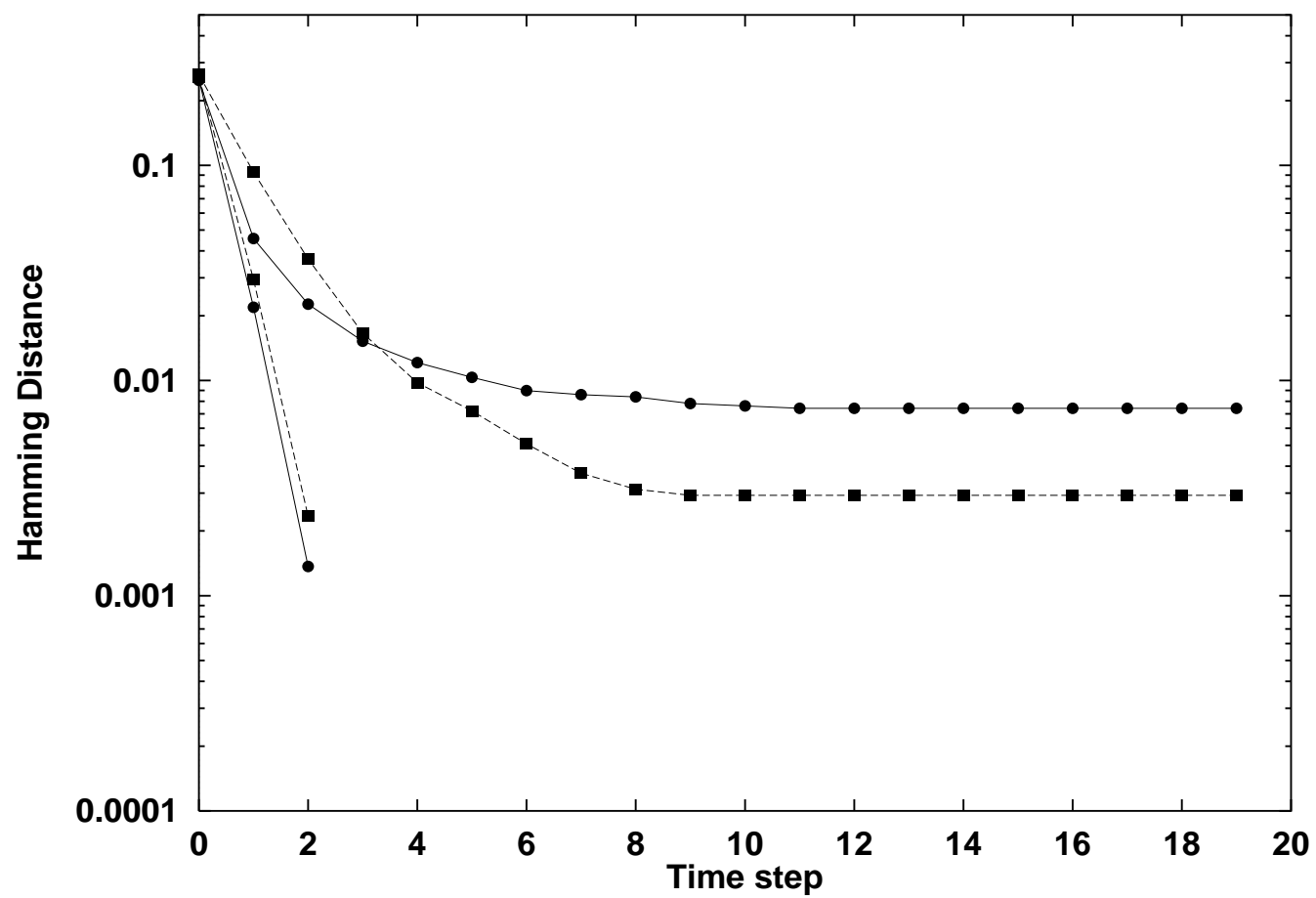

Figure 2: Hamming distance as a function of time for a network of $N=$ 512 neurons. The solid lines represent standard parallel updating while the dotted lines represent the present algorithm. The upper curves are for $P=48$ and the lower curve is for $P=32$. The initial Hamming Distance is 0.25 and all data is averaged over ten sets of patterns with ten patterns from each set. 


\section{Simulating Deterministic Cellular Automata}

For stochastic models, the unreliability of ICMP mentioned above can be simply ignored, however, for deterministic systems, ICMP has to be made reliable in much the same way higher level protocols like TCP make IP reliable.

The model we chose for demonstration purposes is the simple cellular automaton known as Life, which was invented by J.H. Conway.[18] This widely studied automaton is an interesting testing ground because of its myriad properties, including the capability of performing universal computations. 19. In this model, the cells, $C_{i j}$, of a two dimensional square lattice are initially assigned values $C_{i j} \in\{0,1\}$. The cells then change their values in parallel by summing over the states of their nearest neighbors:

$$
C_{i j}(t+1)=H\left(\sum_{m=i-1}^{i+1} \sum_{n=j-1}^{j+1} C_{m n}(t), C_{i j}(t)\right) .
$$

where,

$$
H(x, y)= \begin{cases}1 & \text { if } x-y=3 \\ y & \text { if } x-y=2 \\ 0 & \text { otherwise. }\end{cases}
$$

For this experiment we want to make use of a large number of devices, hence, we need an algorithm that works for validating devices as the number of non-validating devices is much too small. As eq. 6 suggest, the simplest approach is to send out two Echo Request for each cell, asking if $x-y=2$ or $x-y=3$. To do this we copy the value $-w_{1}$ into the first data element of the ICMP message (to counter the type value in the first word), then the nearest neighbor cell values are copied into the next 8 data elements of the ICMP message, followed by a $-2(-3)$ to have the validating device determine if $x-y$ is $2(3)$. If $H(x, y)$ does not evaluate to 1 then the checksum will be invalid and no response will come from the device.

To handle the unreliability of ICMP, we adopt the following procedure. First, out-of-order replies can be readily dealt with, if the time value, $t$, is placed in the Identifier word, $w_{2}$. ( $-t$ is then placed in the Sequence Number, $w_{3}$, word to balance the checksum.) Second, to guard against valid packets being dropped because the datagram was corrupted during transit, the Echo Request packets are first sent out for all cells in the automaton, then the replies are examined. If a given cell has not replied, a second Echo Request is sent. If again it does not reply, then we conclude that the answer is indeed $H(x, y)=0$. (One, could ask a third time, however, we find that two requests are generally sufficient for accurate results.) 
One might worry, that by using the checksum to evaluate eq. 6 we are robbing the checksum of its primary function, namely, detecting errors in transmitted packets. Could for example, a single bit error lead to a false positive? There are 3 factors to be considered: 1) The probability of a single bit error occurring anywhere in a datagram is less than $2^{-20}$. 113 2) Since we are only asking if the checksum evaluates to 2 or 3 , only errors in the 2 least significant bits are important. 3) Of the $2^{8}$ possible configurations of the neighboring cells, only half of them would yield a different value of $H(x, y)$ if a single cell value were changed. Hence, a false positive should occur less than 1 in $2^{24}$ cell updates - which is much more than we can achieve with this algorithm using current networks.

As a first experiment involving the network automaton we created a small $4 \times 4$ automaton and initialized it with a so-called glider[18] - a repeating configuration of cells which move about in the space of the automaton. Then a control automaton, implemented in the conventional fashion on a single processor was given the exact same initial state and the two automata were left to evolve, with a cell-by-cell comparison being made after each automata update. During the simulation time of $2^{17}$ site updates, no deviation from the control automaton was detected. In other words, the simple control procedures explained above were enough to enable our deterministic automaton to run using unreliable ICMP messages.

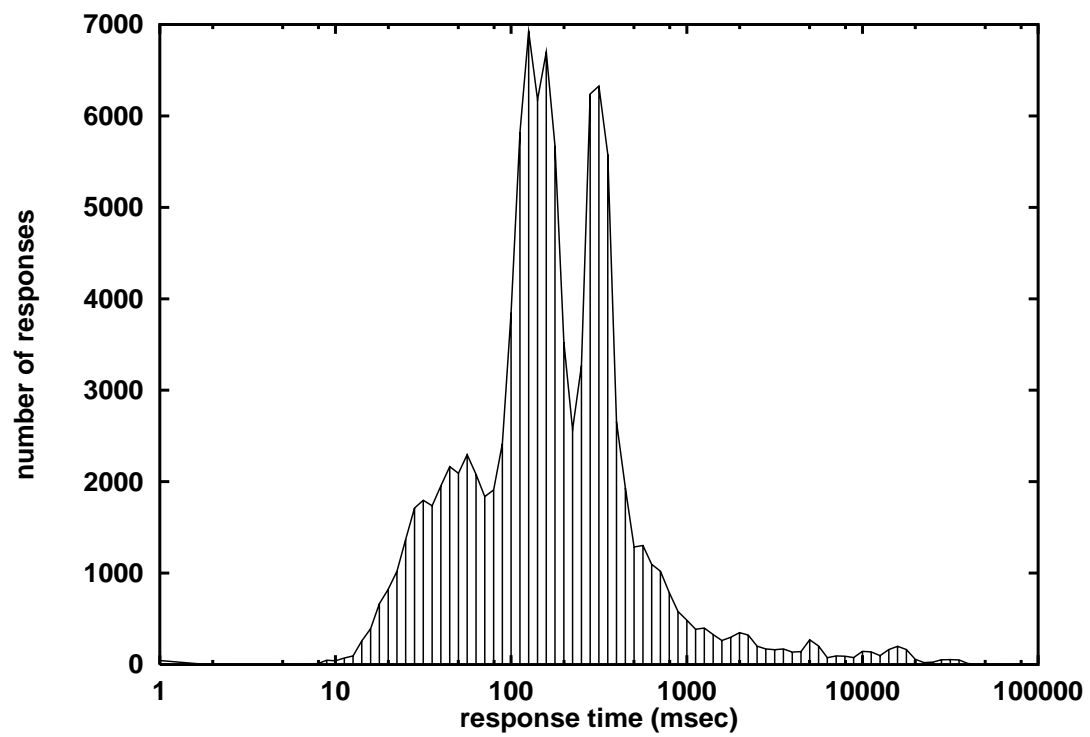

Figure 3: Histogram of average response time for an Echo Request to a randomly chosen Internet device. 
For our final experiment, we attempted to simulate an automaton with $200 \times 500$ cells using $10^{5}$ Internet devices. Although we expected this experiment to be difficult given that our laboratories has only a $1 \mathrm{Mbit} / \mathrm{s}$ connection to the Internet, an unexpected problem we encountered here was the widely varying response times of the different devices. Fig. 3 depicts the average response times for more than $10^{5}$ Internet devices. As can be seen, the response time varies from a few milliseconds to several tens of seconds. In order to use as many of these devices as possible, we first discard all of those with an average response time of more than 10 seconds. Then we order the remainder from slowest to fastest and assign them sequentially to the cells beginning with $(1,1)$. If we then send the requests sequentially to the Internet devices, and delay examining the responses until all the requests have been sent, we can mask the response time of the slowest devices. In this manner we were able to successfully update this very large system for a few time steps. Unfortunately, the Internet is currently not stable enough for simulations of this size and durations, therefore the experiments usually ended after two time steps because the Internet automata developed large deviations from the control automata. 


\section{Discussion}

The experiments described above have proven that the concept of computing using communication is realizable, in other words, it is possible to perform some types of simulations using Internet messaging protocols. Beyond the proof-of-principle demonstrations are questions of performance and efficiency. As was stated in the introduction, communication performance is increasing more rapidly than computational performance. Currently, however, most networks are operating far below state-of-the-art capabilities.

In the example of Conway's Life, we expected that the extra care needed for making the communications reliable would reduce the efficiency of the algorithm. This was compounded by the firewalls set-up to protect our laboratories computers from hackers that reduced our effective bandwidth to the Internet from $100 \mathrm{Mbit} / \mathrm{s}$ to less than $1 \mathrm{Mbit} / \mathrm{s}$. Given that a complete IP/ICMP datagram including the IP header information for our automaton consists of 48 bytes, we would expect to be able to send out a maximum of 2600 messages per second. In reality, we were barely able to sustain a speed of 200 messages per second, meaning it took nearly 2000 seconds to complete one update of our largest system. By contrast, it took our conventional program less than half of one second to update the same system. On our laboratories intranet, it was possible to sustain a speed of approximately 1200 cell updates per second, which is still about 4 orders of magnitude slower than what can be achieved on a single processor.

For the stochastic neural network models, the performance was far better. Given a network of 512 neurons, each datagram, including the IP header, contains about 1056 bytes or 8448 bits, therefore, a theoretical maximum of 11837 datagrams could be sent per second across a $100 \mathrm{Mbit} / \mathrm{s}$ network. Again, in practice, we find it difficult to sustain a rate of more than 1500 datagrams per second - less than 14 percent of the maximum.

Normally, the performance of a neural network model can be measured in terms of the number of coupling updates per seconds (cups). For the conventional parallel updating algorithm we were able to achieve on the SGI O2 workstation used to control our messaging algorithm a speed of $5.5 \cdot 10^{6} \mathrm{cups}$, while the ICMP messaging algorithm itself reaches $0.76 \cdot 10^{6}$ cups. In other words, the messaging algorithm is only approximately 7 times slower than a conventional algorithm running on a single processor. Hence, even though this algorithm is not quite as efficient as an equivalent program running on a single processor, its far more efficient than the deterministic algorithms examined here or by Barabási, et al. [5]

It should be noted, that neither the computer used for these experiments nor the laboratory network represent state-of-the art resources. It is possible 
to obtain single processor computers an order of magnitude faster than the one used in this study, and networks about two orders of magnitude faster. It would be interesting then to repeat these experiments, especially for the stochastic neural network model, on a state-of-the-art network to determine whether or not the performance gap decreases.

Recall, that in the introduction, we talked about developing algorithms to take advantage of idle resources and with respect to this goal, the neural network algorithm is successful. Unlike the network, the host computer can easily sustain an output of much more than 1500 datagrams per second. In order to slow it down to this this speed, the program pauses execution after each packet is sent. During this pause, a timesharing operating system can switch to another processes and perform some useful work before returning to the neural network program.

In conclusion, the approach described herein represents an intriguing alternative for performing parallel, distributed processing and we expect this approach to become more attractive with increasing network bandwidth. Especially in the area of stochastic algorithms, the results generated to date are promising enough to justify continued investigation.

\section{Acknowledgments}

The author would like to thank J. Klaas and F. Zimmermann for many useful discussions related to this work. 


\section{References}

[1] Gordon E. Moore. Cramming more components onto integrated circuits. Electronics, 38(8):35-39, 1965.

[2] Ian Foster. The grid: A new infrastructure for 21st century science. Physics Today, 55:965-974, 2002.

[3] Eric Korpela, Dan Werthimer, David Anderson, Jeff Cobb, , and Matt Lebofsky. Seti@home: Massively distributed computing for seti. Computing in Science and Engineering, 3:78-85, 2001.

[4] Jim Basney and Miron Livny. Deploying a high throughput computing cluster. In Rajkumar Buyya, editor, High Performance Cluster Computing, chapter 5. Prentice Hall, 1999.

[5] Albert-Lászió Barabási, Vincent W. Freeh, Hawoong Jeong, and Jay B. Brockman. Parasitic computing. Nature, 412:894-897, 2001.

[6] T. Berners-Lee, R. Fielding, and H. Frystyk. Hypertext transfer protocol - http/1.0. RFC 1945, Internet Engineering Task Force, 1996.

[7] R. Fielding, J. Gettys, J. Mogul, H. Frystyk, L. Masinter, P. Leach, and T. Berners-Lee. Hypertext transfer protocol - http/1.1. RFC 2616, Internet Engineering Task Force, 1999.

[8] J. Postel. Transmission control protocol. RFC 793, Internet Engineering Task Force, 1981.

[9] J. Postel. Internet protocol. RFC 791, Internet Engineering Task Force, 1981.

[10] K. Nichols, S. Blake, F. Baker, and D. Black. Transmission control protocol. RFC 2474, Internet Engineering Task Force, 1998.

[11] J. Postel. Internet control message protocol. RFC 792, Internet Engineering Task Force, 1981.

[12] R. Braden, D. Borman, and C. Partridge. Computing the internet checksum. RFC 1071, Internet Engineering Task Force, 1988.

[13] C. Partridge J. Stone, M. Greenwald and J. Hughes. Performance of checksums and crcs over real data. IEEE Transactions in Networking, 6:529-543, 1998. 
[14] J. J. Hopfield. Neural networks and physical systems with emergent collective computational abilities. Proceedings of the National Academy of Science, USA, 79:2554-2558, 1982.

[15] G. A. Kohring. Finite-state neural networks. Journal of Statistical Physics, 62:563-576, 1991.

[16] S. Draghici. On the capabilities of neural networks using limited precision weights. Neural Networks, 15:395-414, 2002.

[17] G.A. Kohring. Artificial neurons with arbitrarily complex internal structures. Neurocomputing, 47:103-118, 2002.

[18] M. Gardner. The fantastic combinations of john conway's new solitaire game of "life". Scientific American, 223(4):120-123, 1970.

[19] E. Berlekamp, J. Conway, and R. Guy. Winning Ways for your Mathematical Plays, volume II. Academic Press, London, 1982. 